\title{
Regulation of the MAPK signaling pathway by miR-421-5p in rats under light pollution
}

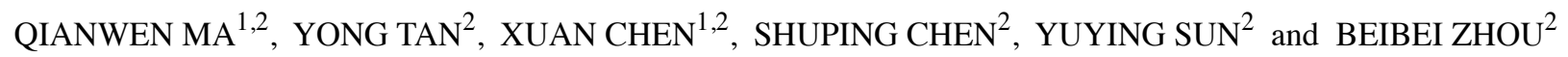 \\ ${ }^{1}$ Zhenjiang Hospital Affiliated to Nanjing University of Chinese Medicine, Zhenjiang Hospital of Traditional Chinese \\ Medicine, Zhenjiang, Jiangsu 212000; ${ }^{2}$ Nanjing University of Chinese Medicine, Nanjing, Jiangsu 210023, P.R. China
}

Received March 13, 2018; Accepted September 11, 2018

DOI: 10.3892/ijmm.2018.3874

\begin{abstract}
The present study aimed to explore the difference in the expression profiles of ovarian microRNA sequences in rats in a light pollution environment and rats in a normal light environment. Rats in the control group were exposed to 12 -h light/dark cycles, while rats in the model group were continuously exposed to 24 -h light. The ovaries were extracted from the two groups of rats, and Illumina HiSeq 2500 high-throughput sequencing technology was used to detect the differences in microRNA (miRNA) expression among the two groups. Fluorescence quantitative reverse transcription-polymerase chain reaction was used to verify the differential expression of miRNA. The present study was designed to experimentally validate the interaction between miR-421-5p and mitogen-activated protein kinase (MAPK) 7 by using the dual-luciferase reporter system, and to explore the expression of proteins in the MAPK signaling pathway with a lentiviral vector-mediated small hairpin RNA interference against microRNA-421-5p. The expression of 45 miRNAs was significantly different. In total, 13 miRNAs were upregulated, of which 5 miRNA sequences were known and 8 were predicted. Furthermore, 32 miRNAs were downregulated, of which 11 miRNA sequences were known and 21 were predicted. The results of the luciferase reporter assay confirmed the targeting association between miR-421-5p and MAPK7. The expression levels of MAPK and genes in its downstream signaling pathways, including c-Fos, CREB and c-Myc, were downregulated when miR-421-5p was overexpressed and upregulated when miR-421-5p was silenced. The
\end{abstract}

Correspondence to: Professor Yong Tan, Nanjing University of Chinese Medicine, 138 Xianlin Avenue, Nanjing, Jiangsu 210023, P.R. China

E-mail:nzyszy@163.com

Abbreviations: ERK, extracellular signal-regulated kinase; GO, Gene Ontology; KEGG, Kyoto Encyclopedia of Genes and Genomes; MAPK, mitogen-activated protein kinase

Key words: microRNA sequencing, ovary, light pollution, mitogen-activated protein kinase signaling pathway, miR-421-5p differential expression of miRNAs may serve an important role in the development of the ovary in a light pollution environment. miR-421-5p may regulate ovarian growth and development by targeting the MAPK signaling pathway in light polluted rat ovaries.

\section{Introduction}

In recent years, the extensive application of artificial light has brought great convenience for people. However, at the same time, it has become a new source of pollution. Light pollution generates a marked adverse effect on the environment and on human life $(1,2)$. There is an increasing population who work under different shift schedules, stay up late or have irregular schedules. This has disrupted the conventional sleep pattern of getting up at sunrise and going to bed at sunset, thus causing certain harm on the human body to a variable extent and increasing the risk of incidence of a series of diseases, as manifested by female reproductive system diseases, such as menstrual disorders, ovulatory disorders, infertilitas feminis, spontaneous abortion and dysmenorrhea $(3,4)$.

MicroRNAs (miRNAs) are a type of non-coding RNA with a length of $22 \mathrm{nt}$, which are extensively present in eukaryotes, such as mammals. miRNAs may cause the degradation of their target messenger RNA (mRNA) or suppress its translation by specific base pairing with the target mRNA, allowing these genes to be regulated post-translationally. As an important mode of post-translational regulation, miRNAs serve an important role in the regulation of the development of numerous diseases, including processes such as cell proliferation and apoptosis, endocrinosity, metabolism, lipid metabolism and neural differentiation (5-11). Numerous studies on important biological processes have reported that a differential expression of miRNAs is present in a variety of diseases. The present study established a continuous light-induced rhythm disturbance rat model to simulate a light polluted environment. The effect of this environmental factor was then examined on rat ovary microRNAs and signaling pathways by analyzing the rat ovary miRNA expression profile via sequencing and the association between miRNA and signaling pathways, thus providing an experimental basis for clinical treatment and prevention. 


\section{Materials and methods}

Experimental animals. Two-month old Sprague Dawley rats (Beijing Wei Tong Li Hua Experimental Animal Technology Co., Ltd., Beijing, China) weighting $200 \pm 20 \mathrm{~g}$ were used for the experiments. Rats were housed at room temperature $\left(22-24^{\circ} \mathrm{C}\right)$ with a humidity of $55-70 \%$, and were provided standard granulated food and water. All experimental protocols involving animals were approved by the Ethics Committee of Zhenjiang Hospital of Traditional Chinese Medicine (Zhenjiang, China).

Reagents and instruments. The reagents and instruments used in the study were as follows: Agilent 2100 Bioanalyzer (Agilent Technologies, Inc., Santa Clara, CA, USA), NanoDrop (Thermo FisherScientific,Inc., Waltham,MA,USA),NEBNext Multiplex Small RNA Library Prep Set for Illumina kit (cat. no. E7300L; New England BioLabs, Inc., Ipswich, MA, USA), TRIzol (cat. no. 15596018; Thermo Fisher Scientific, Inc.), PrimeScript RT-PCR kit (cat. no. RR014A; Takara Biotechnology Co., Ltd., Dalian, China), Premix EX Taq (cat. no. DRR041A; Takara Biotechnology Co., Ltd.), a nucleic acid and protein concentration analyzer (cat. no. SMA4000; Merinton Instrument, Inc., Ann Arbor, MI, USA), 40-W commercially available incandescent lights for daily use (cat. no. E27; Kedao, Hangzhou, China), Olympus CX22 microscope (Olympus Corporation, Tokyo, Japan), Simmah high-precision light intensity detector (Yuan Hengtong, Shenzhen, China), DNA Gel Extraction kit (cat. no. D2500-02; Omega Bio-Tek, Inc., Norcross, GA, USA), T vector (cat. no. D2006; Bi Yun Tian, Shanghai, China), T4 DNA ligase (cat. no. 2011A; Takara Biotechnology Co., Ltd.), Plasmid Extraction kit (cat. no. D6943-01; Omega Bio-Tek, Inc.), restriction endonucleases XhoI (cat. no. 1094; Takara Biotechnology Co., Ltd.) and NotI (cat. no. 1166; Takara Biotechnology Co., Ltd.), Lipofectamine 2000 transfection reagent (cat. no. 11668-027; Thermo Fisher Scientific, Inc.), fetal bovine serum (cat. no. Sh30084.03; Hyclone; GE Healthcare Life Sciences, Logan, UT, USA), RPMI 1640 culture medium (cat. no. 10-040-CRV; Corning Incorporated, Corning, NY, USA), microplate reader (Genios Pro F50; Tecan Group, Ltd., Mannedorf, Switzerland), 293T cells (Mei Xuan, Shanghai, China), Dual-Luciferase Assay kit (cat. no. E1910; Promega Corporation, Madison, WI, USA), rat ovarian luteal granulosa cells (cat. no. 23241; Mei Xuan), pLVshRNA-EGFP (2A) Puro (cat. no VL3103; Ying Mao Sheng Ye, Beijing, China), packaging plasmids $\mathrm{pH} 1$ and $\mathrm{pH} 2$ (Ying Mao Sheng Ye), serum-free Dulbecco's modified Eagle medium (cat. no. 10-040-CRV; Corning Incorporated), polybrene (cat. no. H9268; Sigma-Aldrich; Merck KGaA, Darmstadt, Germany), stripping buffer (cat. no. sw3020; Solarbio Science \& Technology Co., Ltd., Beijing, China), dithiothreitol (cat. no. 233155; Merck KGaA), bovine serum albumin (BSA; cat. no. 735094; Roche Diagnostics, Basel, Switzerland), BCA protein assay kit (cat, no. P0010; Bi Yun Tian), pre-stained protein marker (intermediate molecular weight; cat. no. SM0671; Fermentas; Thermo Fisher Scientific, Inc.), radioimmunoprecipitation assay (RIPA) lysis buffer (cat. no. P0013; Bi Yun Tian), enhanced chemiluminescence (cat. no. WBKLS0500; EMD Millipore, Billerica, MA, USA), Mini-PROTEAN Tetra Cell (cat. no. 165-8001; Bio-Rad Laboratories, Inc., Hercules, CA, USA), MultiSkan3 ELISA reader (Multiskan MK3;
Thermo Fisher Scientific, Inc.), primary antibodies against GAPDH (cat. no. ab8245; Abcam, Cambridge, MA, USA), mitogen-activated protein kinase (MAPK) 7 (cat. no. 12950; Cell Signaling Technology, Inc., Danvers, MA, USA), MYC proto-oncogene (c-Myc; cat. no. ab56; Abcam), Fos proto-oncogene (c-Fos; cat. no. ab134122; Abcam), cAMP responsive element binding protein 1 (CREB; cat. no. 4820; Cell Signaling Technology, Inc.) and phosphorylated (p-) CREB (cat. no. 9198; Cell Signaling Technology, Inc.), and goat anti-rabbit (cat. no. ab6721; Abcam) and mouse (cat. no. ab6789; Abcam) immunoglobulin G (heavy + light chains) conjugated to horseradish peroxidase secondary antibodies.

Experimental methods. A total of 46 female rats were randomly divided into two groups: 23 rats in the control group and 23 rats in the model group. The 23 rats in the control group were exposed to 12 -h light/dark cycles, while the 23 rats in the model group were continuously exposed to 24-h light. The protocols used to generate the experimental rat model of continuous light exposure were based on previously published studies $(12,13)$. The selected light intensity was $300 \pm 20$ lux, and the modeling time was 50 days. We selected $300 \pm 20$ lux in accordance with China's regulations on the ambient illumination and photoperiod indexes for experimental rats (14). The work illumination was 150-300 lux. The criterion for successful modeling was 10 days of continuous keratinized epithelium manifestation of rat vaginal smear. In total, 3 rats did not meet the criterion and were excluded from the experiment, while the remaining 20 rats were assigned as the model group. During the experiment, the rats' estrus cycles, observed by the rats' vaginal smears, were recorded from 9 am to 10 am every morning. The rats were sacrificed at the end of the experiment. Rats were administered an intraperitoneal injection of $10 \%$ chloral hydrate $(350 \mathrm{mg}$ chloral hydrate $/ \mathrm{kg}$ rat body weight) for anesthesia. All rats showed no signs of peritonitis following administration of $10 \%$ chloral hydrate. The abdomen of these rats was immediately opened, and the ovaries were collected, placed in frozen tubes and stored in a refrigerator at $-80^{\circ} \mathrm{C}$ for further analyses.

RNA extraction and quality control. Total RNA was extracted from the ovary tissues obtained from rats of the control and model groups with TRIzol, according to the supplier's instructions. Then, the quality of the RNA was examined using the Agilent 2100 Bioanalyzer. Quality detection was performed in order to avoid contamination by any degradation product and to ensure that the total RNA concentration and integrity met the sequencing requirements. The extracted RNA was stored in a refrigerator at $-80^{\circ} \mathrm{C}$ for further use.

Library construction and sequencing. According to the instructions of the NEBNext Multiplex Small RNA library Prep Set for Illumina kit, $2 \mu \mathrm{g}$ of total RNA (RIN $>8$ ) was used for library construction. The constructed library was purified by PAGE, and 140 bp segments were recovered. Agilent 2100 Bioanalyzer was used to detect the library quality, and Qubit 2.0 Fluorometer was used to quantify the library. Upon mixing the different index marked DNA libraries, 50 bp single-end sequencing was performed, according to the instructions of the 
Illumina HiSeq instrument. The sequence information was read with the HiSeq Control Software + OLB + GAPipeline-1.6 (Illumina) provided in the HiSeq instrument.

Bioinformatics analysis pipeline. miRNA sequencing provided the original data. Next, filtration was performed to remove the contamination and connection sequences, and the length of the determined sequence, reads and data output were then collected and calculated. The sequences obtained were compared with the known miRNAs in the miRBase v20 database, and annotations were performed on known miRNAs. In addition, the sequences were compared with the Rfam database, and analysis was performed to obtain the distribution of non-coding RNAs in the small RNAs. Next, the experimental sequences obtained by sequencing were compared with the complete genome sequence of a specific species, and new miRNAs were predicted with the folding model (15). Subsequently, the differential expression of miRNAs between different samples was analyzed, and clustering model analysis was performed using $\mathrm{R}$ (16).

Analysis of the differential expression of miRNAs. DESeq (v1.18.0) software (17) was used to perform the statistical analysis of the differential expression of known miRNAs in the samples of the two groups. The DESeq results were screened according to the criterion of significant difference (miRNA expression $\geq 2$-fold difference; $\mathrm{P} \leq 0.05$ ), and the upregulation and downregulation of significantly differential expression of miRNAs was calculated.

Gene Ontology (GO) and Kyoto Encyclopedia of Genes and Genomes (KEGG) significance enrichment analysis. GO is an international standard gene classification system. It comprises of three ontologies: Description of a gene's molecular function, cell composition and bioprocesses involved. The main biological function of these differential expression miRNA target genes was determined through the GO function Significance Enrichment Analysis (18). KEGG is the main public database of these pathways. The significance enrichment analysis of KEGG can predict the signaling pathways of significant enrichment in the target genes of differentially expressed miRNAs.

Validation of the differential expression of miRNAs. Fluorescence-based reverse-transcription polymerase chain reaction (RT-qPCR) was used to detect the differential expression of miRNAs, and to validate the results from the high-throughput sequencing. All reactions were performed in a total volume of $20 \mu \mathrm{l}$, using the PrimeScript RT-PCR kit, according to the manufacturer's instructions. Upon termination of the reaction, the quantitative cycle $(\mathrm{Cq})$ values were determined. The mean $\mathrm{Cq}$ value from three replicates was measured, and $\Delta \mathrm{Cq}$ was the difference between the $\mathrm{Cq}$ value of the target gene and the $\mathrm{Cq}$ value of the internal reference. The thermocycling conditions were denaturation at $95^{\circ} \mathrm{C}$ for $30 \mathrm{sec}, 40 \mathrm{cycles}$ of $95^{\circ} \mathrm{C}$ for $5 \mathrm{sec}$ and $60^{\circ} \mathrm{C}$ for $34 \mathrm{sec}$, and end stage $95^{\circ} \mathrm{C}$ for $15 \mathrm{sec}, 60^{\circ} \mathrm{C}$ for $1 \mathrm{~min}$ and $95^{\circ} \mathrm{C}$ for $15 \mathrm{sec}$. Relative fold changes in expression were calculated using the formula $2^{-\Delta \Delta \mathrm{Cq}}(19)$. The ribosomal RNA U6 gene was used as the internal reference (primers, forward TGA
CACGCAAATTCGTGAAGCGTTC and reverse CCAGTC TCAGGGTCCGAGGTATTC). The forward primers for the miRNAS were: miR-344a, GCGGCGGUCAGGCUCCUG GCUA; miR-466b-5p, GGCGGUAUGUGUGUGUGUAU GUC; miR-466c-3p, GCGGCGGUAUACAUGCACACA UAC; and miR-421-5p, CGGCGGGGCCUCAUUAAAUG UUU. The downstream generalized primer sequence is CAG TGCAGGGTCCGAGGTAT.

Dual-luciferase reporter assay. Cryopreserved cells were thawed using the rapid thawing method. Briefly, cells were quickly removed from the $-80^{\circ} \mathrm{C}$ ultra-low temperature freezer, immediately plunged into a $37.5^{\circ} \mathrm{C}$ thermostatic water bath, and rapidly thawed with continuous agitation. The cells were then cultured, and the medium was changed after $\sim 48 \mathrm{~h}$. The cells were cultured until $80 \%$ confluence, and the medium was then removed and $1 \mathrm{ml}$ trypsin was added into the culture flask. The cells were incubated in a $37^{\circ} \mathrm{C}$ incubator for $\sim 30 \mathrm{~min}$ and pipetted into a $25 \mathrm{ml}$ centrifuge tube, followed by centrifugation at $4^{\circ} \mathrm{C}$ and $111.8 \times \mathrm{g}$ for $6 \mathrm{~min}$. Cell debris was collected and $5 \mathrm{ml}$ pre-warmed complete medium was added. The cell suspension was then transferred into a new cell culture flask and cultured in an incubator. Dimethyl sulfoxide:fetal bovine serum:cell culture medium (1:2:7) was mixed to prepare the cell freezing medium. Cells were digested when reaching $100 \%$ confluence and collected in a centrifuge tube. Following centrifugation at $4{ }^{\circ} \mathrm{C}$ and $111.8 \mathrm{x} \mathrm{g}$ for $6 \mathrm{~min}$, the cells were collected and suspended in $1.5 \mathrm{ml}$ freezing medium in a cryotube by gentle pipetting. The cell name, freezing time and operator name were labelled on the tube. A total of $1 \mathrm{ml}$ cell suspension was placed onto a hemocytometer, covered with a cover glass and incubated for $\sim 2$ min until the cell suspension was evenly distributed. Cell numbers were counted under a microscope.

The 3'-untranslated regions (UTRs) of the target gene MAPK7 were designed and manufactured. TargetScan bioinformatics analysis software (7) was employed, which predicted that bases 168-274 at the 3'-UTR of the MAPK7 gene may complementarily pair with microRNA-421-5p. Therefore, 313-nt fragments containing potential complementary binding sites for microRNA-421-5p were designed and synthesized by Sangon Biotech Co., Ltd. (Shanghai, China). Restriction recognition sites for XhoI and NotI were added at the $5^{\prime}$ and $3^{\prime}$ ends of the fragments, respectively. To anneal the MAPK 3'-UTR fragment to form double-stranded DNA, the 3'-UTR sense sequence and 3'-UTR antisense sequence of MAPK7 were dissolved in TE buffer $(\mathrm{pH} 8.0)$ at a final concentration of $100 \mu \mathrm{M}$. The samples were denatured at $95^{\circ} \mathrm{C}$ for $2 \mathrm{~min}$, and the temperature was then reduced at a rate of $1^{\circ} \mathrm{C}$ per $90 \mathrm{sec}$ to $25^{\circ} \mathrm{C}$ and incubated for $30 \mathrm{~min}$. The annealing products were stored at $4^{\circ} \mathrm{C}$ or frozen at $-20^{\circ} \mathrm{C}$ for later use. The products were diluted to $200 \mathrm{nM}$ for the subsequent ligation reaction.

Next, psiCHECK2-MAPK7 3'-UTR was constructed. The vector psiCHECK2 contained restriction sites for XhoI and NotI in its multiple cloning site (MCS), and was therefore linearized by digestion with XhoI and NotI. The psiCHECK2 vector was digested in a $37^{\circ} \mathrm{C}$ water bath for $2 \mathrm{~h}$ and then incubated at $70^{\circ} \mathrm{C}$ for $5 \mathrm{~min}$ to stop the reaction.

DNA products of the double digestion were recovered following agarose gel electrophoresis. The MAPK7 3'-UTR was ligated with the psiCHECK 2 vector. Next, E. coli competent 
cells were prepared and transformed with the recombinant plasmid. Then, recombinant plasmids were extracted for identification of recombinant clones. Subsequently, a dual-luciferase activity assay of the regulation of miR-421-5p on the target gene MAPK7 was performed.

The psiCHECK2 vector is used primarily to detect the activity of the miRNA of interest. In the dual-luciferase assay, the target mRNA of the miRNA is inserted into the MCS downstream of the firefly luciferase reporter gene, which is the major reporter. If the exogenous miRNA binds to the mRNA of the target gene, the expression of the firefly luciferase gene will be inhibited, and the fluorescence value will be reduced. The Renilla luciferase reporter gene is used as an internal reference gene to normalize the results and reduce experimental errors.

In the present study, the two target gene fragments were designed and cloned into the MCS downstream of the firefly luciferase reporter gene in the psiCHECK2 vector. The miRNA, if present, would bind to the mRNA of the firefly luciferase gene to degrade the mRNA and inhibit the transcription and subsequent translation of the firefly luciferase gene, thus resulting in a reduced fluorescence value. By contrast, if the miRNA is absent, the mRNA of the firefly luciferase gene would not be degraded, and its transcription and translation would continue normally. Therefore, the fluorescence value would be higher. To test this hypothesis, transfection of recombinant vectors into 293T cells and dual-luciferase reporter gene activity assay were conducted.

The plasmid psiCHECK 2 was transfected into 293 T cells with Lipofectamine 2000, and luciferase activity was examined with the Promega Dual-Luciferase Assay kit. Experimental groups were as follows: Group 1, miR-421-5p + psiCHECK2-MAPK7; group 2, miR-4 21-5p + psiCHECK2-MAPK7-mutant (mut); group 3, negative miRNA + psiCHECK2-MAPK7; group 4, negative miRNA + psiCHECK2-MAPK7-mut; group 5, miR-421-5p + psiCHECK2; group 6, let7 + EZH1; and group 7, psiCHECK2. Three replicates were analyzed in each group.

Effect of miR-421-5p regulation on ovarian granulosa cells in rats. Rat ovarian luteal granulosa cells were used to examine the effect of miR-421-5p in vitro. Cells in the normal group were not treated, while those in the overexpression, silenced and control groups were transfected with virus overexpressing miR-421-5p, virus with silenced miR-421-5p expression and empty vector control virus, respectively.

Sequences of small hairpin RNA (shRNA) were designed using dedicated software and synthesized in full length for overexpression and silencing. The target fragments mentioned above were ligated into the pLVshRNA-EGFP (2A) Puro vector, and then amplified and sequenced. Lentiviruses were prepared and transfected into host cells. The expression of downstream proteins was detected by western blotting. Samples were processed, and the extracted proteins were mixed with loading buffer and boiled for $10 \mathrm{~min}$. Upon cooling down slowly to room temperature, the samples were centrifuged briefly and stored at $-20^{\circ} \mathrm{C}$ for testing. Then, the proteins were quantified and the protein concentration was determined by the BCA method. Next, western blotting of the target protein and the internal reference protein was performed. In addition, MAPK7, c-Fos, c-Myc and CREB expression in the ovaries of the two groups of rats was also determined by RT-qPCR.
Table I. Estrus cycle comparison of the two rat groups.

\begin{tabular}{lcccc}
\hline Group & Proestrus & Estrus & Metestrus & Diestrus \\
\hline Model $(\mathrm{n}=23)$ & 121 & $115^{\mathrm{a}}$ & $118^{\mathrm{a}}$ & $134^{\mathrm{a}}$ \\
Control $(\mathrm{n}=23)$ & 38 & 428 & 10 & 12 \\
\hline
\end{tabular}

Data are presented as $\mathrm{n} .{ }^{\mathrm{a}} \mathrm{P}<0.05$.

A

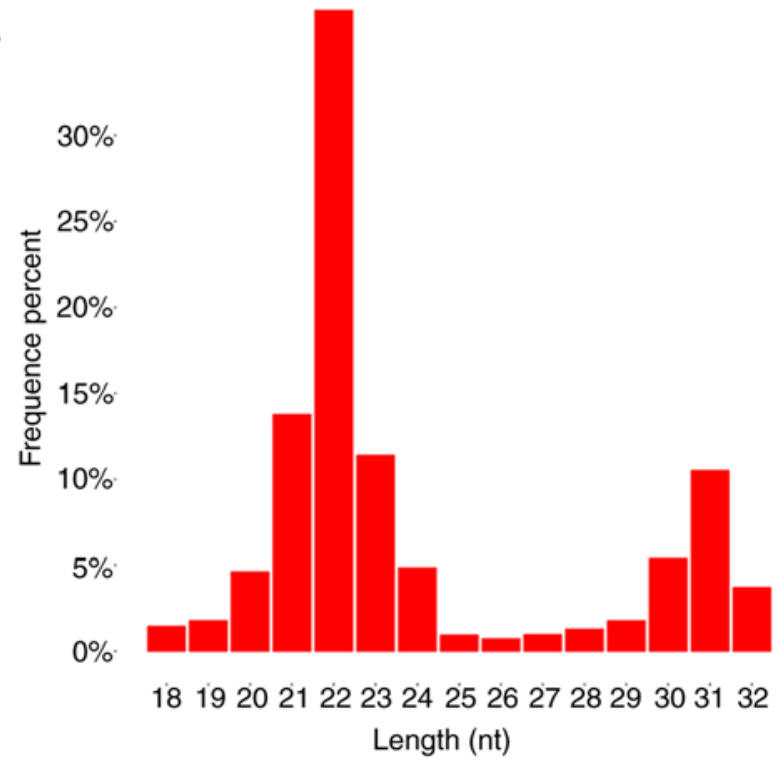

B

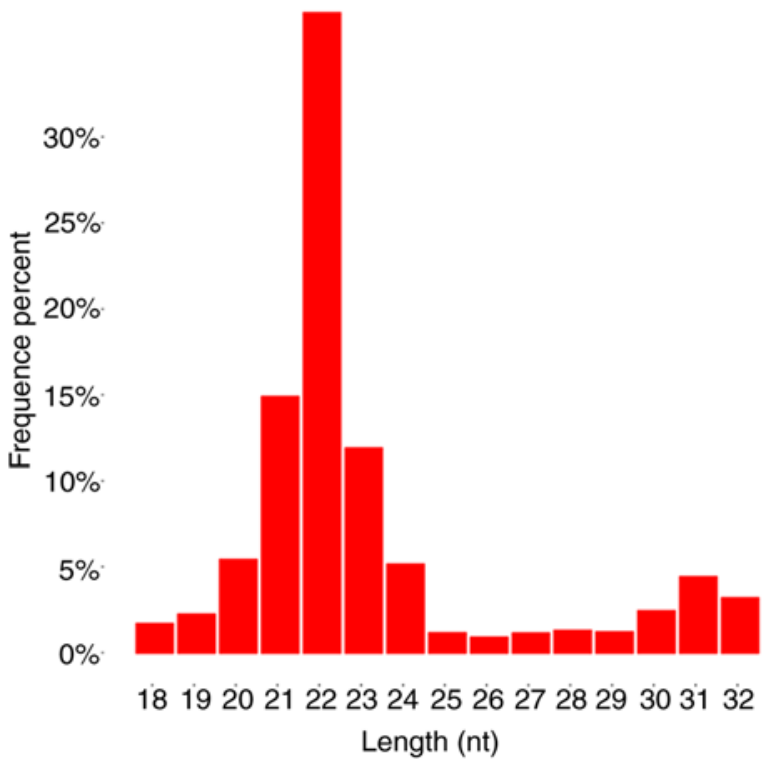

Figure 1. Distribution of ovarian RNA sequences in the (A) control and (B) model group of rats.

Western blotting. Total protein was extracted from samples using RIPA lysis buffer, via boiling for $10 \mathrm{~min}$. Protein quantification experiments were performed via BCA (3). Subsequently, $20 \mu \mathrm{g}$ protein per lane were separated by SDS-PAGE (12\% separation gel and 5\% concentrated gel), and transferred to polyvinylidene difluoride membranes. The membrane was blocked in 3\% BSA with TBS-Tween-20 (TBST) overnight at $4{ }^{\circ} \mathrm{C}$. The membrane was incubated with the 
Table II. Number of reads of small RNAs in the ovaries of the two rat groups.

\begin{tabular}{lcccccr}
\hline Group & rRNA & snoRNA & miRNA & tRNA & snRNA & Others \\
\hline Control & 184,370 & 106,251 & 432,305 & $4,073,512$ & 11,620 & 72,044 \\
Model & 135,613 & 133,589 & 671,118 & $2,789,467$ & 11,777 & 49,077 \\
\hline
\end{tabular}

A
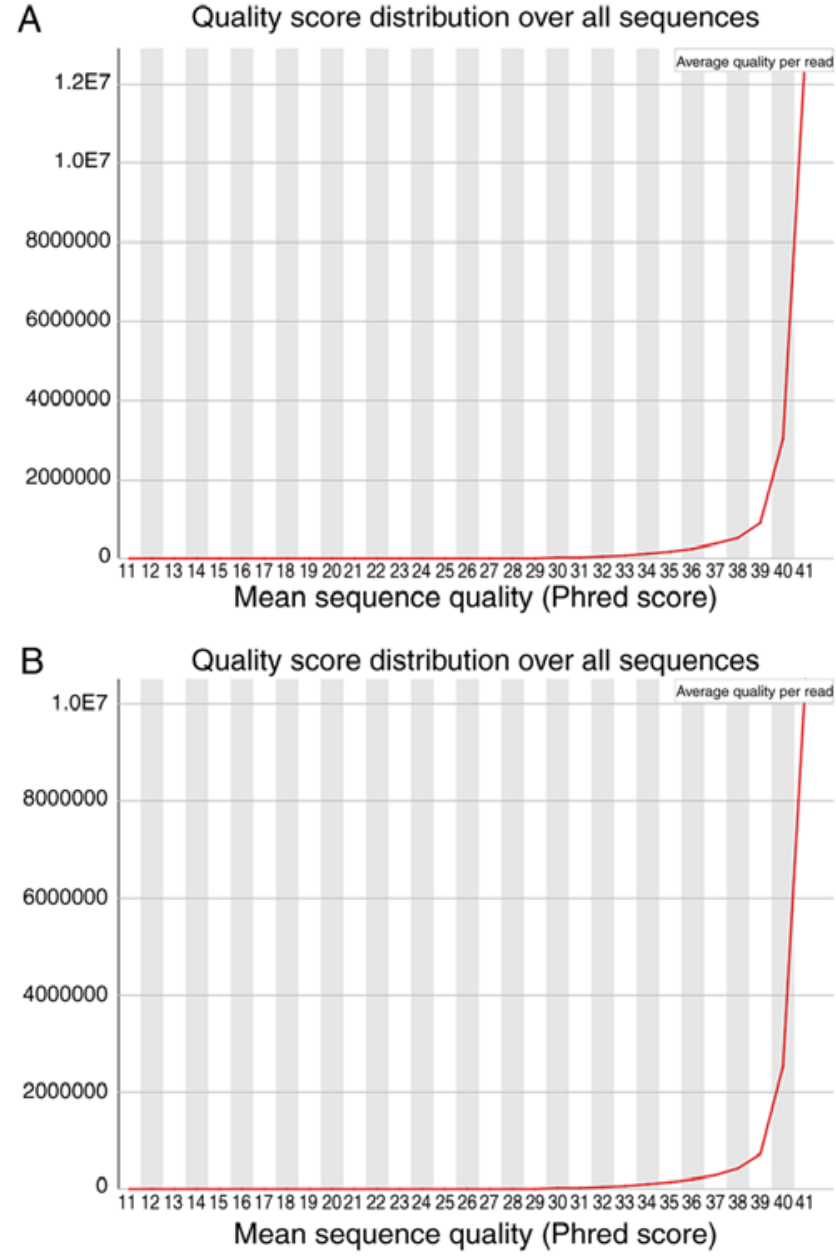

Figure 2. Average distribution of base sequence of (A) control and (B) model rats.

following primary antibodies at room temperature for $30 \mathrm{~min}$ : GAPDH (1:10,000), MAPK7 (1:1,000), c-Myc (1:1,000), c-Fos $(1: 1,000)$, CREB (1:1,000) and p-CREB (1:1,000).

The membrane was then washed with TBST 5 times, each time for $3 \mathrm{~min}$ and incubated with goat anti-rabbit and anti-mouse secondary antibodies (both 1:3,000) at room temperature for 40 mins. Protein bands were visualized using enhanced chemiluminescence. Quantity One software (v.4.6.6; Bio-Rad Laboratories, Inc.) was used for densitometry analysis.

$R T-q P C R$. TRIzol was used for total RNA extraction from ovarian tissue. PrimeScript RT-PCR kit and Premix EX Taq were applied for the RT and $\mathrm{APCR}$ reactions, respectively. Primers were designed by Sangon Biotech Co., Ltd. RT was conducted in accordance with the manufacturer's protocol for
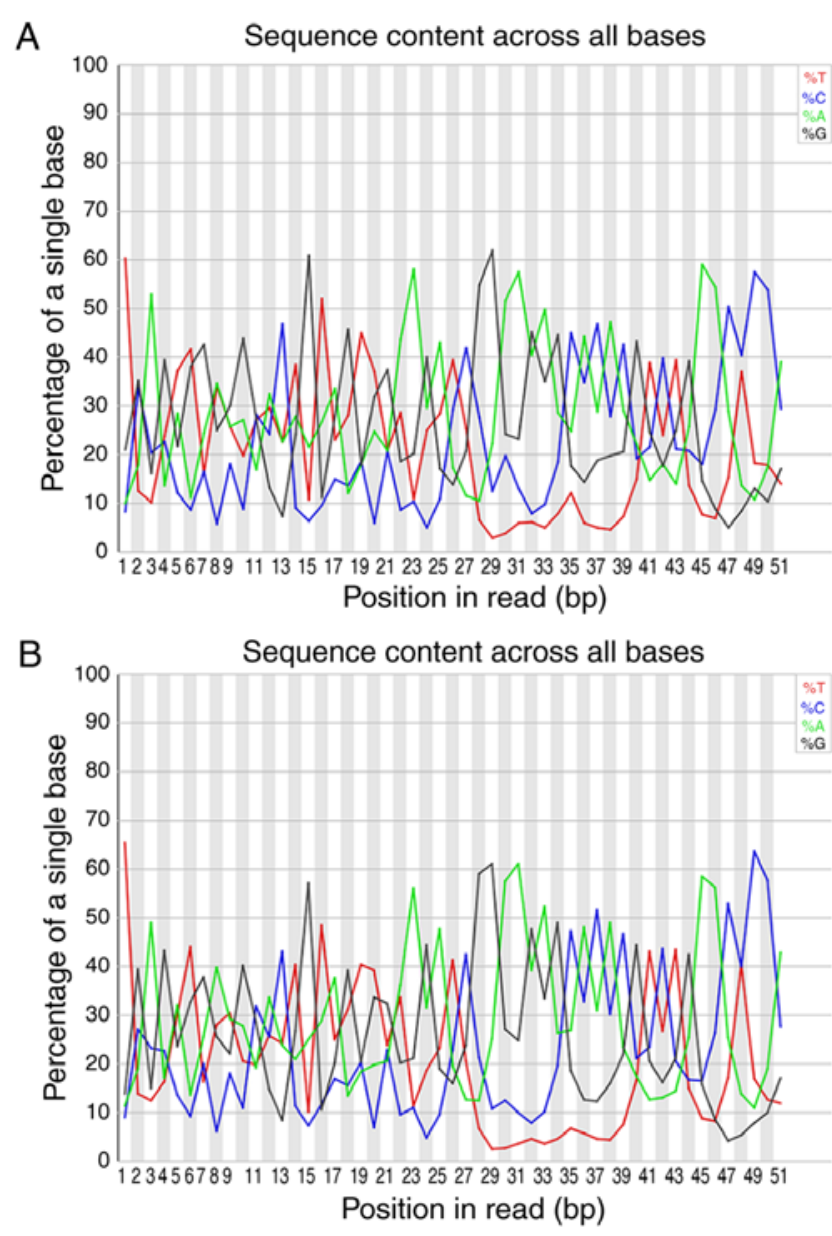

Figure 3. Distribution of GC base content of (A) control and (B) model rats.

the PrimeScript RT-PCR kit.qPCR was subsequently performed with the following thermocycling conditions: Denaturation at $95^{\circ} \mathrm{C}$ for $30 \mathrm{sec}, 40 \mathrm{cycles}$ of $95^{\circ} \mathrm{C}$ for $5 \mathrm{sec}$ and $60^{\circ} \mathrm{C}$ for $34 \mathrm{sec}$, and end stage $95^{\circ} \mathrm{C}$ for $15 \mathrm{sec}, 60^{\circ} \mathrm{C}$ for $1 \mathrm{~min}$ and $95^{\circ} \mathrm{C}$ for $15 \mathrm{sec}$. The following primer sequences were used: MAPK7 forward, 5'-GCTCCTTCGACGTGACCTTT-3' and reverse, 5'-TCC AGTACCACGTAGACAGA-3'; C-MYC forward, 5'-TGGAGT GAGAAGGGCTTTGC-3' and reverse, 5'-GTGAGAAGTGTC TGCCCGTT-3'; C-FOS forward, 5'-GGGAGCTGACAGATA CGCTC-3' and reverse, 5'-TCAAGTCCAGGGAGGTCACA-3'; CREB forward, 5'-CCCCAGCACTTCCTACACAG-3' and reverse, 5'-TTAAGCACTGCCACTCTGTTC-3'; and GADPH forward, 5'-AGTGCCAGCCTCGTCTCATA-3' and reverse, 5'-GGTGATGGGTTTCCCGTTGA-3'. mRNA levels were quantified using the $2^{-\Delta \Delta \mathrm{Cq}}$ method.

Statistical analysis. SPSS 24.0 software was used for data processing (IBM Corp., Armonk, NY, USA) (20). Differences 
Table III. Differential expression of known microRNAs.

Expression quantity of microRNAs

\begin{tabular}{lccl}
\cline { 2 - 3 } MicroRNA & Control group & Model group & Expression change \\
\hline miR-421-5p & 0 & 211.5 & Upregulated \\
miR-326-5p & 0 & 124.5 & Upregulated \\
miR-132-5p & $2,517.7$ & 388.8 & Downregulated \\
miR-146b-5p & $26,466.9$ & $5,636.8$ & Downregulated \\
miR-466c-3p & 451.1 & 87.5 & Downregulated \\
miR-212-5p & 409.1 & 49.1 & Downregulated \\
miR-466b-5p & 51.0 & 0 & Downregulated \\
miR-21-5p & $1,527,937.3$ & $46,7145.7$ & Downregulated \\
miR-208a-5p & 5.6 & 83.6 & Upregulated \\
miR-6329 & $1,116.7$ & 536.8 & Downregulated \\
miR-338-5p & 133.7 & 362.4 & Upregulated \\
miR-96-5p & 903.7 & 388.9 & Downregulated \\
miR-344a & 26.3 & 0 & Downregulated \\
miR-31a-5p & $5,469.7$ & $2,043.8$ & Downregulated \\
miR-541-5p & 38.3 & 144.8 & Upregulated \\
miR-877 & 57.4 & 6.3 & Downregulated \\
\hline
\end{tabular}

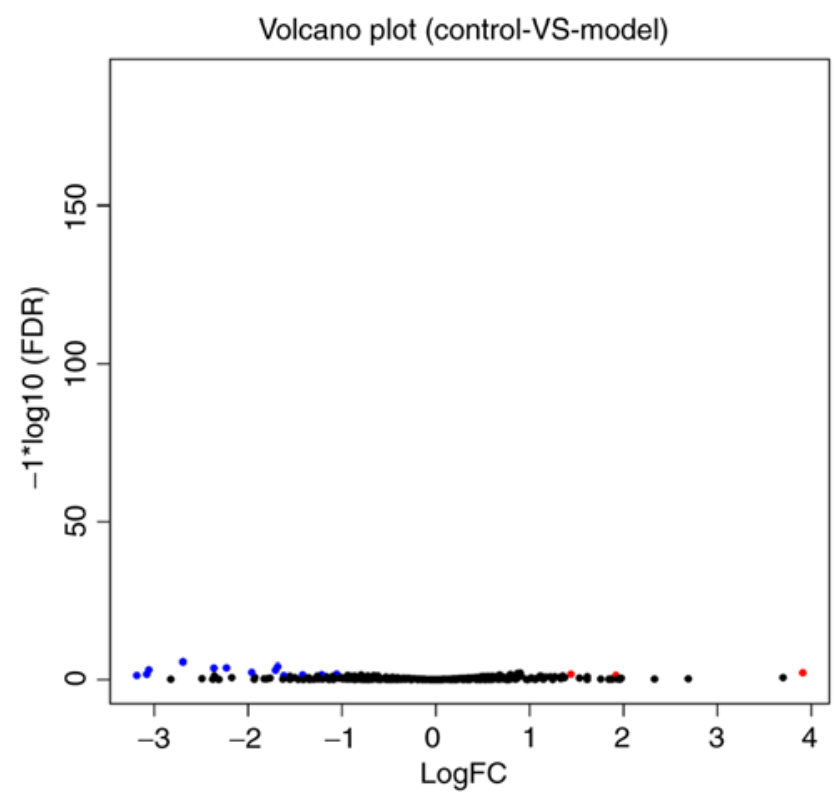

Figure 4. In the differential microRNA volcano plot for significantly differentially expressed microRNAs, the red color represents upregulated expression, while the blue color represents downregulated expression. The $\mathrm{X}$-axis is the fold-change in expression for microRNAs in different samples, while the $\mathrm{Y}$-axis is the statistical significance of the difference in the expression change of microRNAs. The dot image of plus or minus infinity is not shown.

between two groups were examined with the t-test. Differences between multiple groups were examined with one-way analysis of variance for those that complied with a homogeneity of variance post hoc test. Non-parametric test was adopted for those that did not comply with homogeneity of variance. $\mathrm{P}<0.05$ was considered to indicate a statistically significant difference.

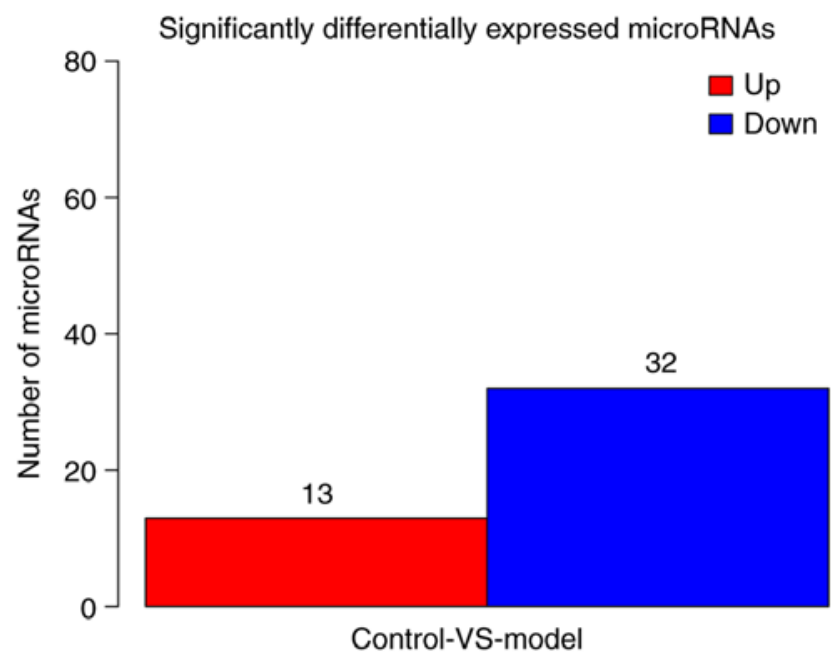

Figure 5. Comparison of microRNA expression in the control and model groups.

\section{Results}

Rat estrus cycles. The number of rats in the control group at the stage of proestrus, metestrus and diestrus was higher compared with the model group (Table I). The number of estrous period in the model group was higher compared with the control group (Table I). These findings suggest that light pollution may lead to disorders of the estrus cycle in rats, prolong the estrus period and shorten the stages of proestrus, metestrus and diestrus.

High-throughput sequencing data. Upon data processing of the original sequences, the number of small RNA sequences was counted in the two groups of rat ovary samples, 
Table IV. Unknown differentially expressed microRNAs.

Expression quantity of microRNAs

\begin{tabular}{lccc} 
MicroRNA & Control group & Model group & Expression change \\
\hline NovelmiRNA-117 & $1,026.7$ & 319.1 & Downregulated \\
NovelmiRNA-118 & 0 & 241.5 & Upregulated \\
NovelmiRNA-122 & 0 & 317.3 & Upregulated \\
NovelmiRNA-131 & 451.1 & 87.5 & Downregulated \\
NovelmiRNA-132 & $2,885.9$ & 0 & Downregulated \\
NovelmiRNA-135 & 388.8 & 0 & Downregulated \\
NovelmiRNA-14 & 34.22 & 0 & Downregulated \\
NovelmiRNA-140 & 137.2 & 44.6 & Downregulated \\
NovelmiRNA-17 & 872.0 & 0 & Downregulated \\
NovelmiRNA-198 & 215.5 & 0 & Downregulated \\
NovelmiRNA-256 & 196.8 & 0 & Downregulated \\
NovelmiRNA-259 & 0 & $7,559.6$ & Upregulated \\
NovelmiRNA-276 & 0 & 266.3 & Upregulated \\
NovelmiRNA-318 & 0 & 0 & Upregulated \\
NovelmiRNA-327 & 879.4 & 0 & Downregulated \\
NovelmiRNA-338 & 902.6 & 0 & Downregulated \\
NovelmiRNA-34 & $346,855.7$ & 0 & Downregulated \\
NovelmiRNA-348 & 25.1 & 0 & Downregulated \\
NovelmiRNA-405 & 78.5 & 0 & Downregulated \\
NovelmiRNA-406 & 258.4 & 0 & Downregulated \\
NovelmiRNA-446 & $14,8717.1$ & 422.6 & Downregulated \\
NovelmiRNA-483 & $1,646.4$ & 9.6 & Downregulated \\
NovelmiRNA-495 & 81.6 & $412,320.7$ & Downregulated \\
NovelmiRNA-512 & 0 & 0 & Upregulated \\
NovelmiRNA-58 & 197.1 & 414.7 & Downregulated \\
NovelmiRNA-60 & 0 & 0 & Upregulated \\
NovelmiRNA-62 & $2,104.2$ & 0 & Downregulated \\
NovelmiRNA-71 & 0 & & Upregulated \\
NovelmiRNA-91 & $2,104.2$ & Downregulated \\
\hline & & 09.3 &
\end{tabular}

and those suitable for analysis contained 1,6733,978 and $1,3724,423$ reads, respectively. The statistical analysis of the distribution of sequences with a length of 18-32 nt revealed that the reads of the obtained sequences of $18-32 \mathrm{nt}$ mainly corresponded to 20-24 nt and reached a peak at 22 nt (Fig. 1A and B), which met the characteristic length of microRNAs. The present results demonstrated that the sequencing data were accurate and reliable, and could be used in subsequent bioinformatics analyses.

Classification and annotation of small RNA sequences. The comparison of sequences with the rat genome revealed that rat samples in the model group totally matched those of rat genome sequences that had 3,790,640 reads, while rat samples in the control group totally matched the rat genome sequences that had 4,880,102 reads. For comparison of these matched sequences with various types of small RNAs, the rat mRNAs in the Rfam database were used, since they provided the number of reads of different types of small RNA (Table II).
Average distribution of base sequences. In the average distribution of sample base sequences, the $\mathrm{X}$-axis corresponds to the average quality value of the base sequence, while the $\mathrm{Y}$-axis is the number of sequences. Fig. 2A and B represent the average quality peak value for the majority of base sequences, which was $>30$ in general, suggesting a good quality of the sequences.

Distribution of GC base content. The GC base content test determines any separation of AT or GC. The X-axis is the base position of the reads, while the Y-axis is the \% of a single base. Different colors represent different base types. It can be observed in Fig. 3A and B that the sample GC base content was balanced and there was no separation in the AT or GC base pairs.

Analysis of the differential expression of miRNAs. When comparing the control with the model group, the expression of 45 miRNAs was significantly different. Among these 45 miRNAs, 13 were upregulated, of which 5 miRNA sequences were known and 8 were predicted. Furthermore, 32 miRNAs 


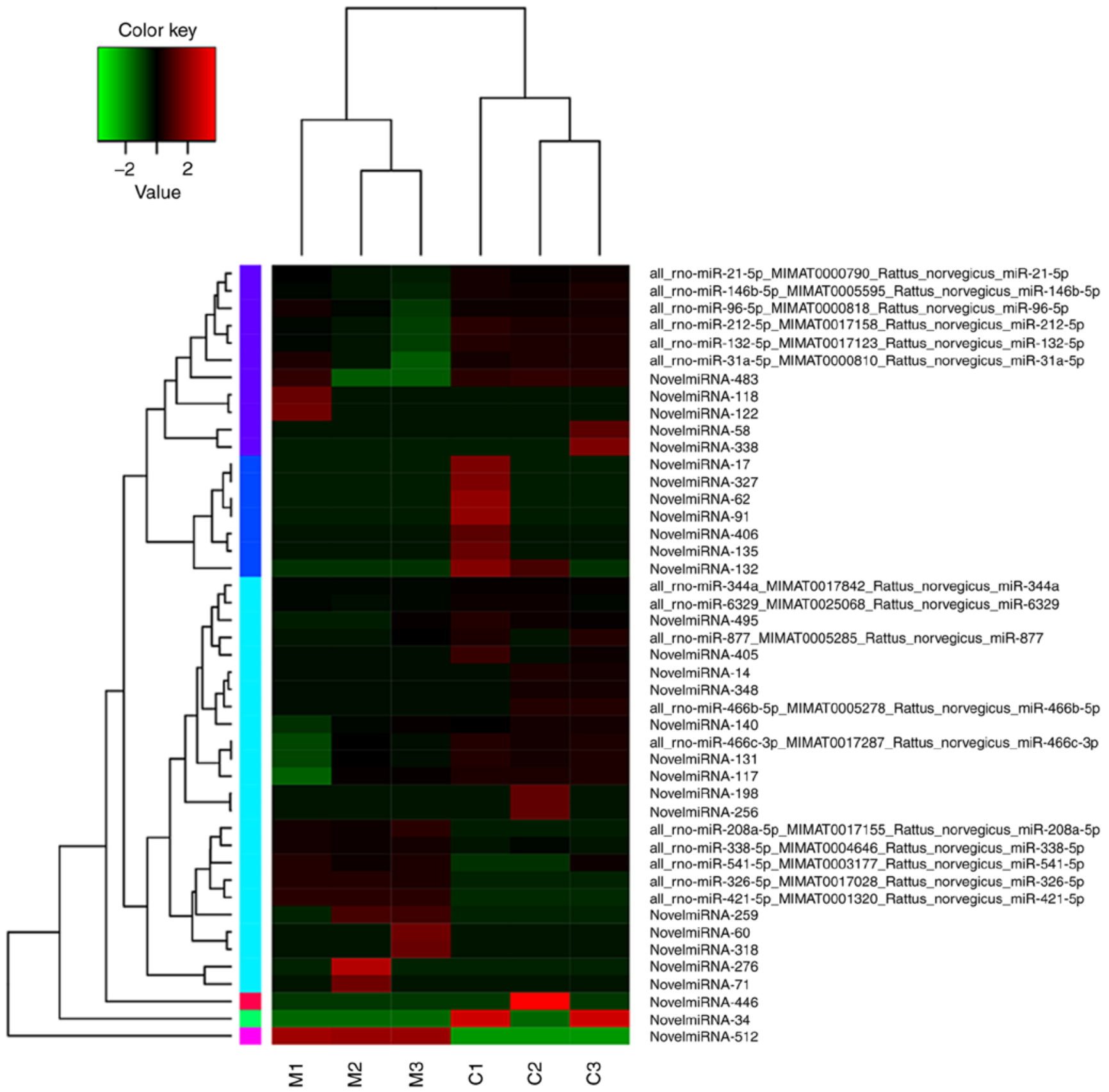

Figure 6. In the differential microRNA expression clusplot for clustering log2 TPM values, the red color represents high expression of microRNAs whereas the green color represents low expression of microRNAs.

were downregulated, of which 11 miRNA sequences were known and 21 were predicted (Tables III and IV).

The differential miRNA volcano plot for significantly differentially expressed miRNAs is shown in Fig. 4. The comparison of miRNA expression in the control and model groups is shown in Fig. 5. The differential miRNA clusplot for clustering $\log 2$ TPM (TPM=microRNA reads $\times 10^{6} /$ total reads) values is shown in Fig. 6. The red color represents high expression of miRNAs and the green color represents low expression of miRNAs. Cluster analysis was used to calculate the similarity of the data and to classify them accordingly in order to cluster miRNAs with the same function or a close association. In this manner, it may be possible to recognize the function of miRNAs and deduce whether they participate in the same metabolic process or cell pathways. Regions with different colors represent different cluster information. Patterns expressed by miRNAs in the same group are similar and may have similar functions or participate in the same biological process.

GO and KEGG function significant differences. Representation of the differential expression of miRNA target genes in the GO enrichment bar graph displayed the count distribution of differential miRNA target genes in a biological process, cell composition and molecular function-enriched GO enrichment. GO analysis also revealed that the samples were mainly enriched in biological regulation, the process of growth and molecular binding in terms of enriched molecular functions 


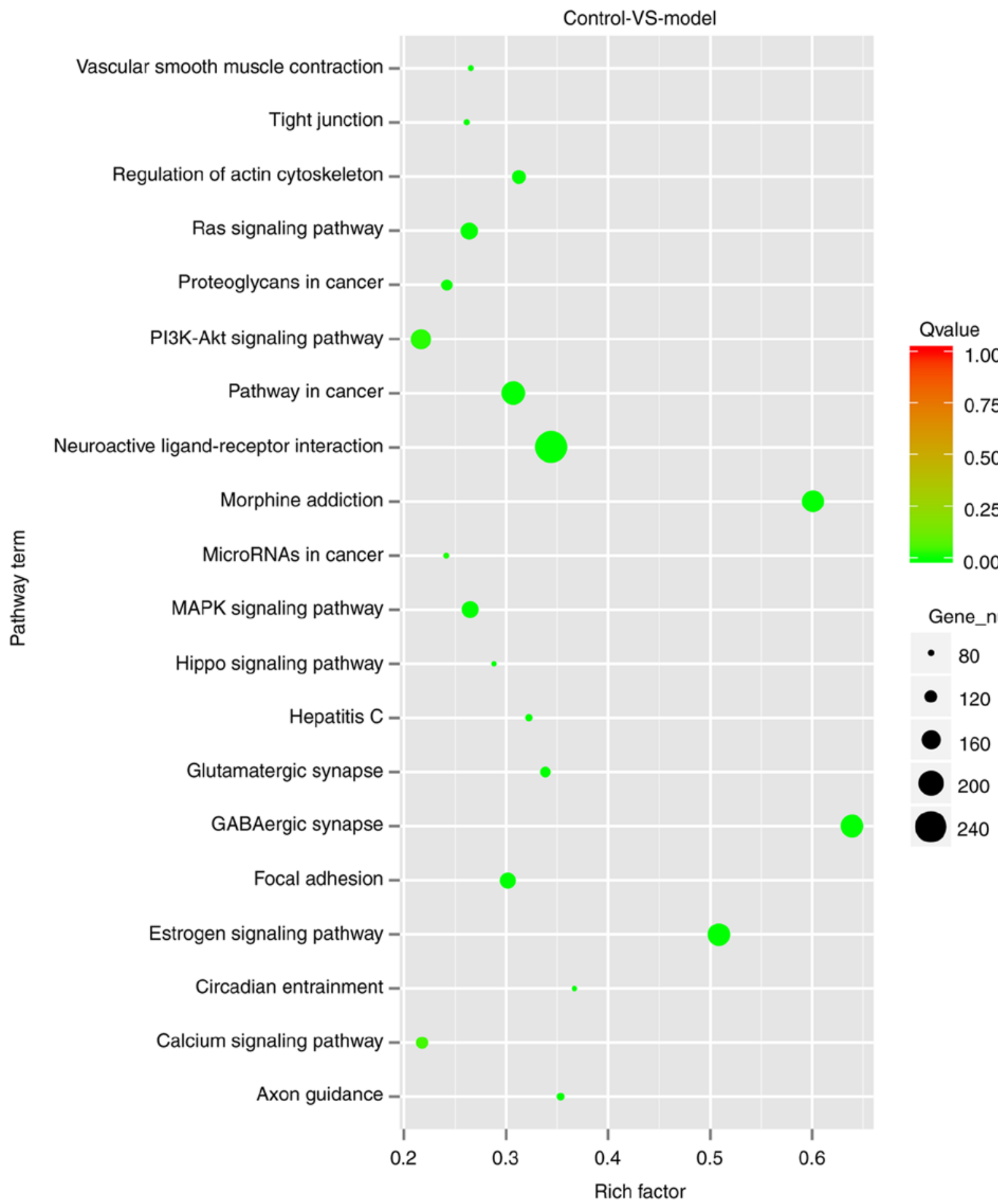

Figure 7. GO enrichment histogram. The $\mathrm{X}$-axis is the enrichment of GO term and the $\mathrm{Y}$-axis is the number of different microRNA target genes in this term. Different colors are used to distinguish biological processes, cell components and molecular functions. GO, Gene Ontology.

(Fig. 7). Scatter plots are used as a method of graphically demonstrating the KEGG enrichment analysis results. In this graph, KEGG enrichment level is measured using the rich factor, also known as Q-value, as well as the number of genes enriched in this pathway. The rich factor refers to the ratio of the number of genes located in this pathway term versus the differentially expressed miRNA target genes from the all annotated genes. The greater the rich factor, the higher the enrichment level. The Q-value refers to the P-value upon correction by the multiple hypothesis testing, which ranges from 0 to 1 . The closer to 0 this value is, the more significant the enrichment becomes. KEGG Pathway Enrichment Analysis revealed that pathways involving miRNA-regulated target genes were mainly enriched in the interaction of nerve tissue receptors, cancer, estrogen and MAPK-associated signaling pathways (Fig. 8).

Validation by $R T-q P C R$. Four significantly differentially expressed miRNAs (miR-344a, miR-466b-5p, miR-466c-3p and miR-421-5p) were validated by RT-qPCR. The results 


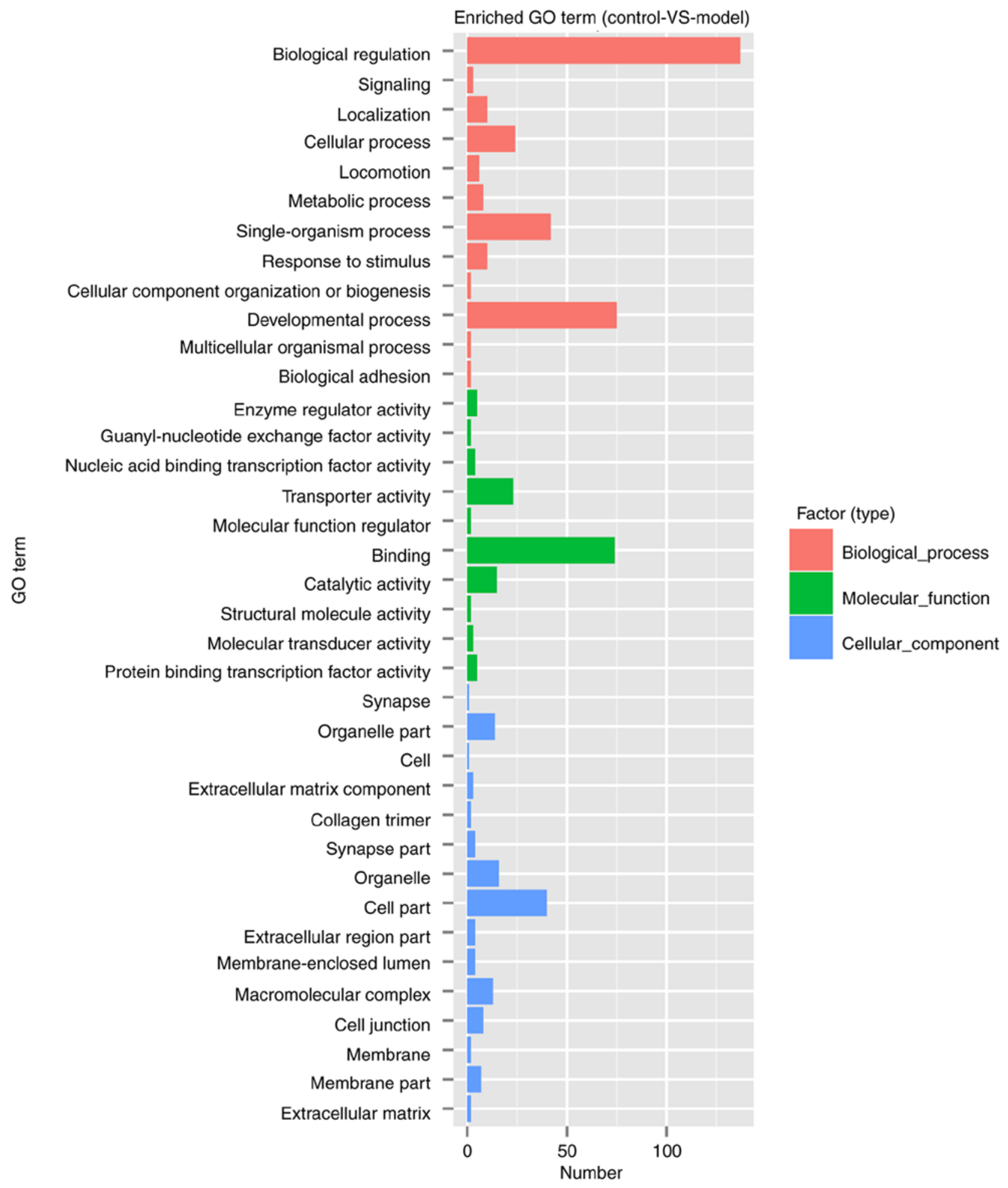

Figure 8. Different microRNA target genes of the Kyoto Encyclopedia of Genes and Genomes rich scatter plot. The Y-axis is the pathway name, while the $\mathrm{X}$-axis shows the rich factor. The size of the points shows the number of differentially expressed microRNA target genes in this pathway, while the color of the points corresponds to the $\mathrm{Q}$-value range.

revealed that the expression changes of the above four microRNAs were consistent with the results from the high-throughput sequencing, further corroborating the accuracy of the sequencing results (Fig. 9).

Dual-luciferase reporter gene assay. The comparison of the fluorescence activity of different carrier plasmids is displayed in Table V. The silencing efficiency of let7 on the EZH1 gene in the positive control group (group 6) was $65.62 \%$, while that of the control group was within the range of allowable error, thus suggesting that these experimental data were reliable. The silencing efficiency of miR-421-5p on MAPK7 gene was $56.36 \%$. The present results suggest that miR-421-5p exhibited good regulation effects on MAPK7. 
Table V. Comparison of fluorescence activity of the carrier plasmid in each group.

\begin{tabular}{lccrr}
\hline Group & $\begin{array}{c}\text { REN/FIR } \\
\text { mean value }\end{array}$ & $\begin{array}{c}\text { Standard } \\
\text { deviation }\end{array}$ & $\begin{array}{c}\text { Residual fluorescence activity } \\
\text { compared with group 7 (\%) }\end{array}$ & $\begin{array}{c}\text { Fluorescence } \\
\text { reduction }(\%)\end{array}$ \\
\hline 1 & 0.259295 & 0.019407 & 43.64 & 56.36 \\
2 & 0.564539 & 0.051092 & 95.01 & 4.99 \\
3 & 0.582167 & 0.013456 & 97.98 & 2.02 \\
4 & 0.602215 & 0.059465 & 101.35 & -1.35 \\
5 & 0.556680 & 0.045377 & 93.69 & 6.31 \\
6 & 0.204253 & 0.027100 & 34.38 & 65.62 \\
7 & 0.594164 & 0.035435 & 100.00 & 0.613 \\
\hline
\end{tabular}

P-values are included for differences in each group vs. group 7. REN, Renilla luciferase; FIR, firefly luciferase.

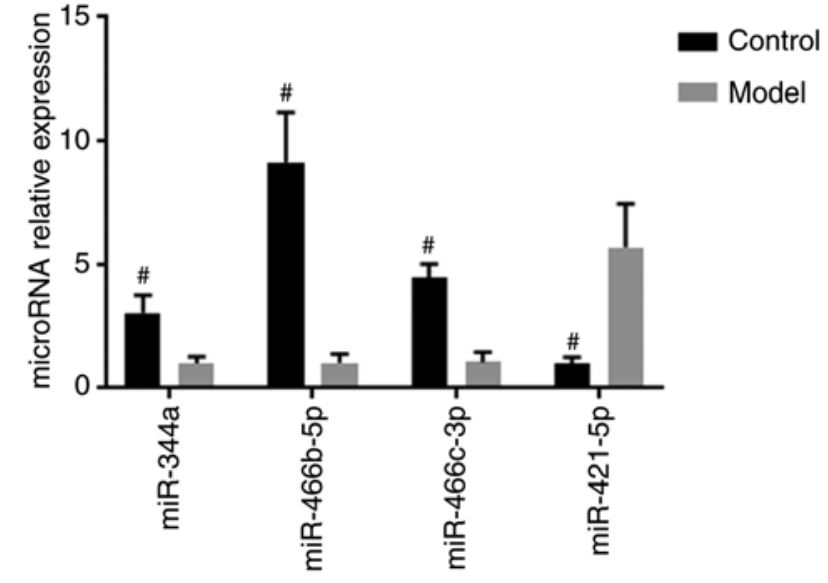

Figure 9. Relative expression levels of four microRNAs in the control and model groups. " $\mathrm{P}<0.05$.

Effect of miR-421-5p on target protein expression in rat ovarian granulosa cells. Rat ovarian granulosa cells were transduced with lentiviral constructs to either silence or overexpress miR-421-5p. Then, the protein expression levels of MAPK7, c-Fos, c-Myc, CREB and p-CREB were assessed by western blotting (Fig. 10)

MAPK7 protein expression in the overexpression group was considerably lower compared with the normal, silenced and control groups $(\mathrm{P}<0.05)$. MAPK7 protein expression in the silenced group was higher compared with the normal and control groups $(\mathrm{P}<0.05)$.

c-Fos protein expression in the overexpression group was markedly lower compared with the normal, silenced and control groups $(\mathrm{P}<0.05)$. c-Fos protein expression in the silenced group was higher compared with the normal and control groups $(\mathrm{P}<0.05)$. c-Myc protein expression in the overexpression group was markedly lower compared with the normal, silenced and control groups $(\mathrm{P}<0.05)$. c-Myc protein expression in the silenced group was higher compare with the normal and control groups $(\mathrm{P}<0.05)$.

CREB protein expression in the overexpression group was markedly lower compared with the normal, silenced and control groups $(\mathrm{P}<0.05)$. CREB protein expression in the silenced group was higher compared with the normal and control groups $(\mathrm{P}<0.05)$. The protein expression of $\mathrm{p}-\mathrm{CREB}$ in the overexpression group was remarkably lower compared with the normal, silenced and control groups $(\mathrm{P}<0.05)$. The protein expression of $\mathrm{p}-\mathrm{CREB}$ in the silenced group was higher compared with the normal and control groups $(\mathrm{P}<0.05)$.

Expression of ovarian MAPK7, c-Fos, c-Myc and CREB, as detected by $R T-q P C R$. The expression of ovarian MAPK7 in the control group was significantly higher compared with the model group ( $\mathrm{P}<0.05$; Fig. 11). The expression of ovarian $\mathrm{c}-\mathrm{Fos}$ in the control group was significantly higher compared with the model group $(\mathrm{P}<0.05$; Fig. 11). The expression of ovarian c-Myc in the control group was significantly higher compared with the model group ( $\mathrm{P}<0.05$; Fig. 11). The expression of ovarian CREB in the control group was significantly higher compared with the model group $(\mathrm{P}<0.05$; Fig. 11).

\section{Discussion}

In recent years, the extensive application of artificial light has brought great convenience in people's life. However, prolonged night-time illumination may extend the exposure time and intensity of the human body to light, which may cause endocrine disorders, circadian rhythm disorders and reproductive dysfunction. It has been reported in the literature that night-shift and shift-schedule workers have increased incidence of diseases such as menstrual disorders and infertilitas feminis. Increased illumination over time or over intensity could cause hypothalamic-pituitary-ovarian axis abnormalities, and affect human life and health $(21,22)$.

The present study combines high-throughput techniques with bioinformatics to discuss light pollution-induced differential expression of miRNAs and possible target genes, and further hypothesizes the mode of action of miRNAs in a light-polluted environment. The results revealed that, among the 45 miRNAs exhibiting highly differential expression in the light-polluted group, 13 were upregulated and 32 were downregulated. Furthermore, the RT-qPCR results confirmed the miRNA expression changes to be consistent with the high-throughput sequencing.

The target genes of differentially expressed miRNAs obtained in the present study are mainly enriched in processes such as biological regulation and growth in terms 

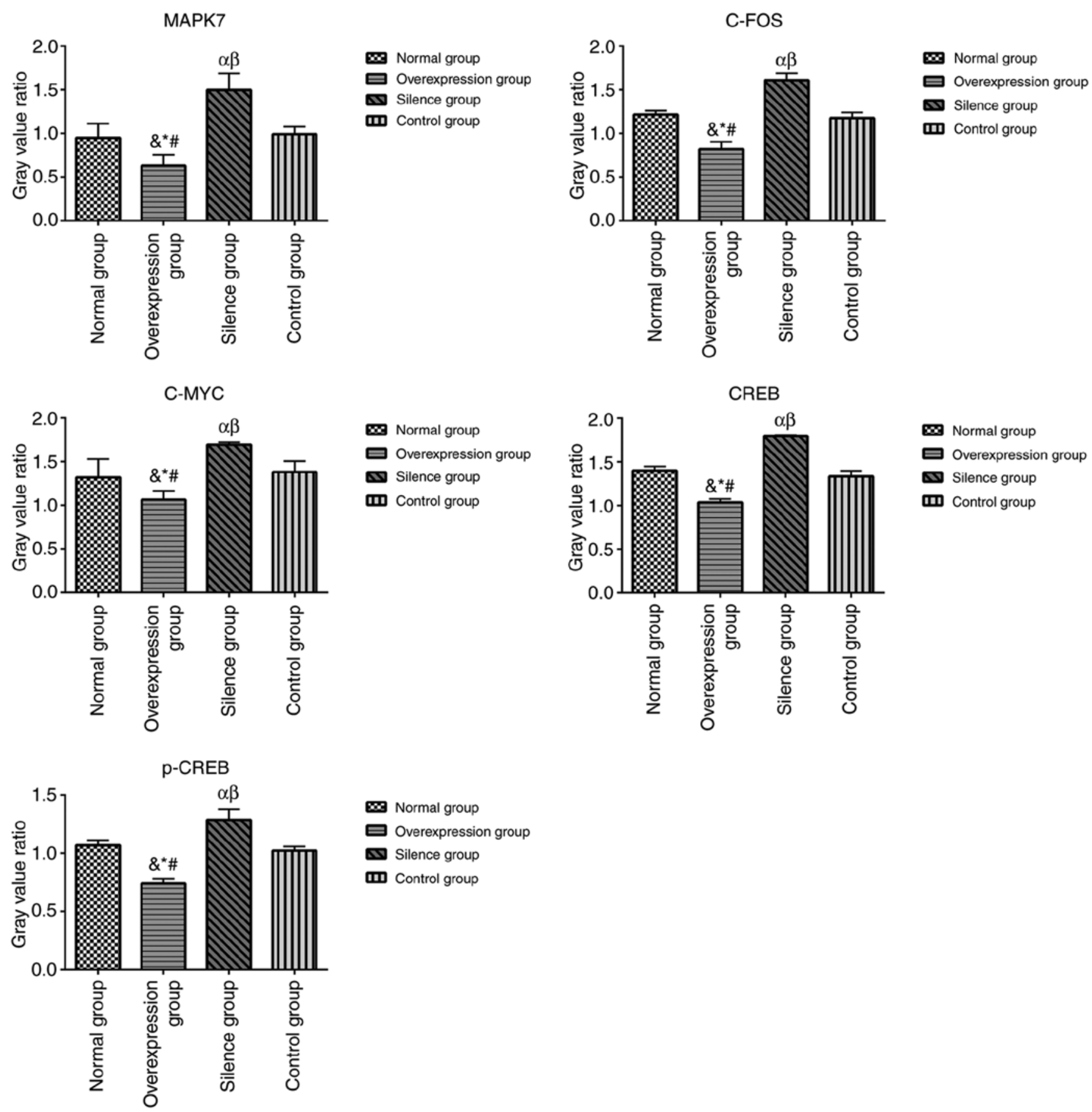

Figure 10. Protein expression levels were determined by western blotting in rat ovarian granulosa cells, following overexpression or silencing of miR-421-5p. Untreated cells were the normal group. Cells transduced with empty vector were the control group. ${ }^{\circledR} \mathrm{P}<0.05$, overexpression group vs. normal group; ${ }^{*} \mathrm{P}<0.05$, overexpression group vs. silenced group; ${ }^{*} \mathrm{P}<0.05$, overexpression group vs. control group; ${ }^{a} \mathrm{P}<0.05$, normal group vs. silenced group; ${ }^{\beta} \mathrm{P}<0.05$, silenced group vs. control group. MAPK, mitogen-activated protein kinase; c-Fos, Fos proto-oncogene; c-Myc, MYC proto-oncogene; CREB, cAMP responsive element binding protein $1 ; \mathrm{p}-$, phosphorylated.

of GO enriched biological functions; in ionic binding in terms of molecular functions; and in the synthesis and metabolism of cell parts, macromolecular compounds and the cell wall in terms of cell composition. KEGG pathway enrichment analysis revealed that pathways involved in miRNA-regulated target genes in light-polluted rats are mainly enriched in cancer, estrogen and MAPK-associated signaling pathways.

miRNAs, a class of non-coding short RNAs, serve an important regulatory role in cell growth and development, and have become a hot spot in the field of biological cytology. The MAPK family is an important factor in the signaling pathways of cell growth and proliferation. MAPKs are a protein kinase family widely distributed in the cytoplasm, with phosphorylation capacity on both threonine and serine (23-25). Extracellular-regulated protein kinases (ERKs) are the first type of MAPKs identified. MAPK7, also known as ERK5, is a member of the MAPK family and is expressed in a variety of tissues. MAPK7 can be regulated by growth factors, cytokines and oxidative stress (26-29), and regulates numerous 
Ovarian MAPK
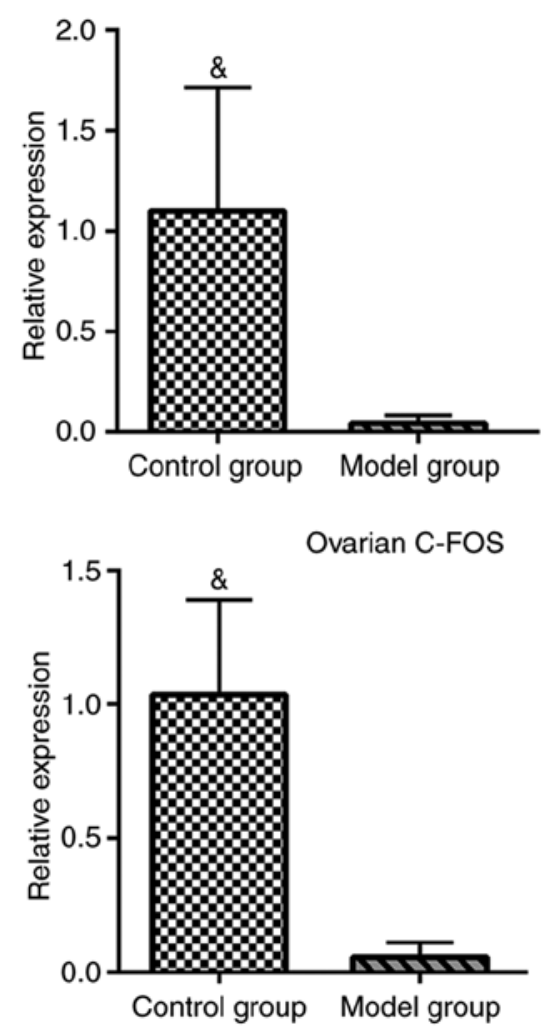

Ovarian C-MYC
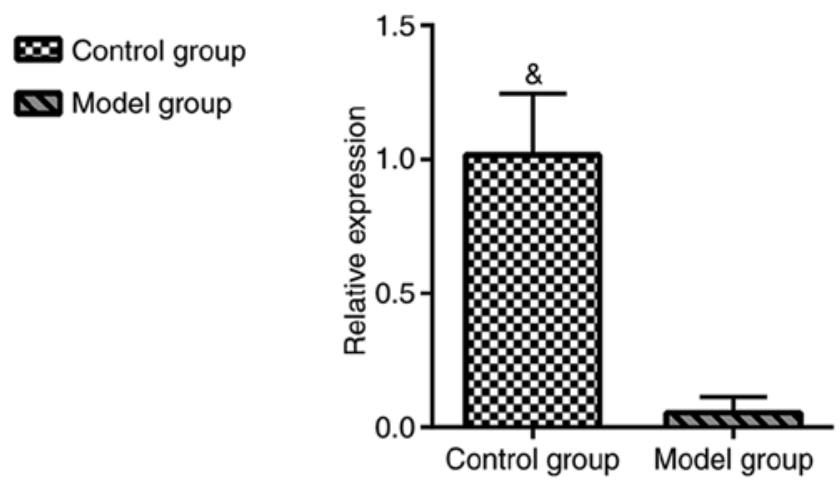
$\mathbf{\infty}$ Control group
$\mathbf{\nabla}$ Model group
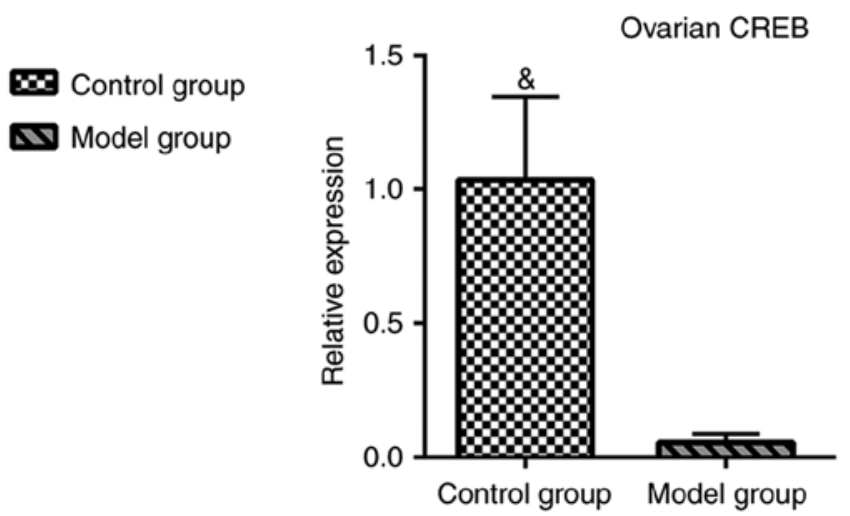

Figure 11. mRNA expression levels of target genes in the ovarian tissues of the control and model rats. ${ }^{\&} \mathrm{P}<0.05$. MAPK, mitogen-activated protein kinase; c-Fos, Fos proto-oncogene; c-Myc, MYC proto-oncogene; CREB, cAMP responsive element binding protein 1.

biological processes, including cell growth, differentiation and apoptosis (30-32).

In the present study, a bioinformatics software was used to predict that miR-421-5p was able to complementarily pair with MAPK7 3'-UTR. Then, a eukaryotic expression vector encoding MAPK7 with miR-421-5p was successfully constructed and confirmed by restriction endonuclease digestion and sequencing. The dual-luciferase reporter assay system revealed that the relative luciferase activity of the wild-type + miR-421-5p group was significantly reduced compared with the wild-type + miRNA internal reference group, suggesting that miR-421-5p directly interacted with the MAPK7 3'-UTR and inhibited the activity of fluorescein. The present study therefore demonstrated that miR-421-5p was involved in the gene regulation of MAPK7 by binding to its 3'-UTR.

In the present study, a lentiviral expression system was used as an interference expression vector to obtain a stable and reliable gene expression alteration. Granulosa cells are closely associated to reproductive endocrine than thecal cells, and miR-421-5p expression was detected in granulosa cells in the early stage. Thus, we selected granulosa cells and not thecal cells for studying the effects of miR-421-5p on the MAPK signaling pathway. The morphology of rat granulosa cells was not significantly changed upon transduction with the lentiviruses, and the cells grew as expected. Thus, cells with miRNA overexpression or knockdown were generated and used to detect the expression levels of the target proteins MAPK7, c-Fos, c-Myc, CREB and p-CREB.
MAPK7 is an important member of the MAPK signaling pathway, and changes in its expression directly affect the signal transduction cascade. MAPK7 mainly affects the function of the ERK signaling pathway in the MAPK signaling pathway, and the ERK pathway serves a decisive role in cell proliferation and differentiation (33-35). In the present study, the expression of MAPK7, c-Fos, c-Myc and CREB, as well as the phosphorylation of CREB, were analyzed by western blotting once miR-421-5p was overexpressed or silenced. Protein phosphorylation is the most basic and important mechanism to regulate and control the activity and function of proteins. The present results revealed that the expression levels of MAPK7, c-Fos, c-Myc, CREB and p-CREB were decreased when miR-421-5p was overexpressed. The ERK signaling pathway mainly affects the proliferation and differentiation of cells, and the expression levels of MAPK7, c-Fos, c-Myc, CREB and p-CREB were increased when miR-421-5p was silenced.

In the experimental observation of the animals, it was remarked that the rats in the 24-h light group did not show sleep deprivation within the exposure time (50 days), and the rats still exhibited normal sleep under the 24-h light condition. Therefore, the conclusions of the present study were caused by continuous light exposure, rather than sleep deprivation.

In summary, miR-421-5p was demonstrated to bind to MAPK7 3'-UTR, inhibit the expression of MAPK7, and significantly reduce the expression of MAPK7, c-Fos, c-Myc, CREB and $p-C R E B$, indicating that miR-421-5p can target the MAPK signaling pathway. These results, together with previous experiments, indicate that light pollution may interfere 
with ovarian growth and development, and this may be mediated by miR-421-5p targeting the MAPK signaling pathway. The effects and mechanisms of continuous light exposure on the hypothalamus and hypophysis will be explored in future studies.

\section{Acknowledgements}

Not applicable.

\section{Funding}

The present study was supported by the Natural Science Foundation of China (grant no. 81373683) and Zhenjiang Health and Family Planning Science Technology Key Project (grant no. SHW2017016).

\section{Availability of data and materials}

The analyzed datasets generated during the study are available from the corresponding author on reasonable request.

\section{Authors' contributions}

QM and YT conceived and supervised the study, and designed the experiments. SC, YS and BZ performed the experiments. $\mathrm{XC}$ analyzed the data. QM wrote the manuscript and YT conducted manuscript revisions. All authors reviewed the results and approved the final version of the manuscript.

\section{Ethics approval and consent to participate}

All experimental protocols involving animals were approved by the Ethics Committee of Zhenjiang Hospital of Traditional Chinese Medicine (Zhenjiang, China).

\section{Patient consent for publication}

Not applicable.

\section{Competing interests}

The authors declare that they have no conflict of interest.

\section{References}

1. Gamble KL, Resuehr D and Johnson CH: Shift work and circadian dysregulation of reproduction. Front Endocrinol (Lausanne) 4: 92, 2013.

2. Dumont $M$ and Paquet J: Progressive decrease of melatonin production over consecutive days of simulated night work. Chronobiol Int 31: 1231-1238, 2014.

3. Bonmati-Carrion MA, Arguelles-Prieto R, Martinez-Madrid MJ, Reiter R, Hardeland R, Rol MA and Madrid JA: Protecting the melatonin rhythm through circadian healthy light exposure. Int J Mol Sci 15: 23448-23500, 2014.

4. Dibner $C$ and Gachon F: Circadian dysfunction and obesity: Is leptin the missing link? Cell Metab 22: 359-360, 2015.

5. Cheng CJ, Bahal R, Babar IA, Pincus Z, Barrera F, Liu C, Svoronos A, Braddock DT, Glazer PM, Engelman DM, et al: MicroRNA silencing for cancer therapy targeted to the tumour microenvironment. Nature 518: 107-110, 2015.

6. Ye D, Guo S, Al-Sadi R and MaTY: MicroRNAregulation of intestinal epithelial tight junction permeability. Gastroenterology 141: 1323-1333, 2011 .
7. Agarwal V, Bell GW, Nam JW and Bartel DP: Predicting effective microRNA target sites in mammalian mRNAs. Elife 4, 2015.

8. Zhou X, Zhu W, Li H, Wen W, Cheng W, Wang F, Wu Y, Qi L, Fan Y, Chen Y, et al: Diagnostic value of a plasma microRNA signature in gastric cancer: A microRNA expression analysis. Sci Rep 5: 11251, 2015.

9. Liu S, da Cunha AP, Rezende RM, Cialic R, Wei Z, Bry L, Comstock LE, Gandhi R and Weiner HL: The host shapes the gut microbiota via fecal microRNA. Cell Host Microbe 19: 32-43, 2016.

10. Kim YS, Kim HR, Kim H, Yang SC, Park M, Yoon JA, Lim HJ, Hong SH, DeMayo FJ, Lydon JP, et al: Deficiency in DGCR8-dependent canonical microRNAs causes infertility due to multiple abnormalities during uterine development in mice. Sci Rep 6: 20242, 2016.

11. Ahmed K, LaPierre MP, Gasser E, Denzler R, Yang Y, Rulicke T, Kero J, Latreille $M$ and Stoffel M: Loss of microRNA-7a2 induces hypogonadotropic hypogonadism and infertility. J Clin Invest 127: 1061-1074, 2017.

12. Zhang YJ, Ma QW and Tan Y: The research of continous illumination on reproductive endocrine of rats and the intervention effect of nourishing Yin and tonifying Yang Fang sequential traditional Chinese medicine. J Nanjing Univ Tradit Chin Med 33: 386-390, 2017.

13. Chen WJ, Sheng WJ, Guo YH and Tan Y: The influence of interfered circadian rhythm on pregnancy and neonatal rats. Sheng Li Xue Bao 67: 521-526, 2015.

14. Wang GZ, Du YJ and Tong J: Circadian rhythms and light responses of clock gene and arylalkylamine $\mathrm{N}$-acetyltransferase gene expressions in the pineal gland of rats Sheng Li Xue Bao 57: 97-102, 2005.

15. Svoboda P and Di Care A: Hairpin RNA: A secondary structure of primary importance. Cell Mol Life Sci 63: 901-908, 2006.

16. $\mathrm{R}$ Core Team: A language and environment for statistical computing. Computing 1: 12-21, 2013.

17. Anders S and Huber W: Differential expression of RNA-Seq data at the gene level-the DESeq package. EMBL, 2012. http:// citeseerx.ist.psu.edu/viewdoc/summary?doi=10.1.1.367.758.

18. Gupta PK, Yoshida R, Imoto S, Yamaguchi R and Miyano S: Statistical absolute evaluation of gene ontology terms with gene expression data international symposium on bioinformatics research and applications. Springer, Berlin, Heidelberg 146-157, 2007.

19. Livak KJ and Schmittgen TD: Analysis of relative gene expression data using real-time quantitative PCR and the 2(-Delta Delta C(T)) Method. Methods 25: 402-408, 2001.

20. Carter V, Jain T, James J, Cornwall M, Aldrich A and de Heer HD: The 3-m backwards walk and retrospective falls: Diagnostic accuracy of a novel clinical measure. J Geriatr Physl Ther 1: 2017.

21. Itani $\mathrm{O}$ and Kaneita Y: The association between shift work and health: A review. Sleep Biol Rhythms 14: 231-239, 2016.

22. Brum MC, Filho FF, Schnorr CC, Bottega GB and Rodrigues TC: Shift work and its association with metabolic disorders. Diabetol Metab Syndr 7: 45, 2015.

23. Sun Y, Liu WZ, Liu T, Feng X, Yang N and Zhou HF: Signaling pathway of MAPK/ERK in cell proliferation, differentiation, migration, senescence and apoptosis. J Recept Signal Transduct Res 35: 600-604, 2015.

24. Guo Z, Kang S, Chen D, Wu Q, Wang S, Xie W, Zhu X, Baxter SW, Zhou X, Jurat-Fuentes JL and Zhang Y: MAPK signaling pathway alters expression of midgut ALP and ABCC genes and causes resistance to Bacillus thuringiensis Cryl Ac toxin in diamondback moth. PLoS Genet 11: e1005124, 2015.

25. He X, Wei Z, Zhou E, Chen L, Kou J, Wang J and Yang Z: Baicalein attenuates inflammatory responses by suppressing TLR4 mediated NF-kappaB and MAPK signaling pathways in LPS-induced mastitis in mice. Int Immunopharmacol 28: 470-476, 2015.

26. Elkins JM, Wang J, Deng X, Pattison MJ, Arthur JS, Erazo T, Gomez N, Lizcano JM, Gray NS and Knapp S: X-ray crystal structure of ERK5 (MAPK7) in complex with a specific inhibitor. J Med Chem 56: 4413-4421, 2013.

27. Javaid S, Zhang J, Smolen GA, Yu M, Wittner BS, Singh A, Arora KS, Madden MW, Desai R, Zubrowski MJ, et al: MAPK7 regulates EMT features and modulates the generation of CTCs. Mol Cancer Res 13: 934-943, 2015.

28. Gavine PR, Wang M, Yu D, Hu E, Huang C, Xia J, Su X, Fan J, Zhang T, Ye Q, et al: Identification and validation of dysregulated MAPK7 (ERK5) as a novel oncogenic target in squamous cell lung and esophageal carcinoma. BMC Cancer 15: 454, 2015. 
29. Lochhead PA, Clark J, Wang LZ, Gilmour L, Squires M, Gilley R, Foxton C, Newell DR, Wedge SR and Cook SJ: Tumor cells with KRAS or BRAF mutations or ERK5/MAPK7 amplification are not addicted to ERK5 activity for cell proliferation. Cell Cycle 15: 506-518, 2016.

30. Tesser-Gamba F, Lopes LJ, Petrilli AS and Toledo SR: MAPK7 gene controls proliferation, migration and cell invasion in osteosarcoma. Mol Carcinog 55: 1700-1713, 2016.

31. Jin M, Wu Y, Wang J, Chen J, Huang Y, Rao J and Feng C: MicroRNA-24 promotes 3T3-L1 adipocyte differentiation by directly targeting the MAPK7 signaling. Biochem Biophys Res Commun 474: 76-82, 2016.

32. Dong X, Lv B, Li Y, Cheng Q, Su C and Yin G: MiR-143 regulates the proliferation and migration of osteosarcoma cells through targeting MAPK7. Arch Biochem Biophys 630: 47-53, 2017.
33. Song X, Wei Z and Shaikh ZA: Requirement of ERalpha and basal activities of EGFR and Src kinase in Cd-induced activation of MAPK/ERK pathway in human breast cancer MCF-7 cells. Toxicol Appl Pharmacol 287: 26-34, 2015.

34. Dai J, Wang T, Wang W, Zhang S, Liao Y and Chen J: Role of MAPK7 in cell proliferation and metastasis in ovarian cancer. Int J Clin Exp Pathol 8: 10444-10451, 2015.

35. Wang D, Wang D, Wang N, Long Z and Ren X: Long non-coding RNA BANCR promotes endometrial cancer cell proliferation and invasion by regulating MMP2 and MMP1 via ERK/MAPK signaling pathway. Cell Physiol Biochem 40: 644-656, 2016.

(8) This work is licensed under a Creative Commons Attribution-NonCommercial-NoDerivatives 4.0 International (CC BY-NC-ND 4.0) License. 